\title{
Evaluation of European Deposit Insurance Scheme Funding Based on Risk Analysis ${ }^{1}$
}

Gómez Fernández-Aguado, Pilar

Trigo Martínez, Eduardo

Moreno Ruiz, Rafael

Partal Ureña, Antonio

IFABS 2021 Virtual Conference

$15 / 09 / 2021$

This work has been financed by I Plan Propio De Investigación, Transferencia y Divulgación Cientifica, Universidad de Málaga, Campus de Excelencia Internacional Andalucía Tech 


\section{Índice I}

\section{(1) Introduction}

(2) Data and sample

(3) EDIS loss distribution

(4) Risk-based premiums

\section{(5) Conclusions}


Context of our work:

- 2008-2009. Financial crisis

- 2014. Directive on Deposit Guarantee schemes (DGSD)

- 2015. EBA proposal for the establishment of European Deposit Insurance Scheme (EDIS)

- <2020: Reinsurance stage. Contribution based on national banking system

- 2020-2024: Coinsurance stage. Contribution based on Banking Union (BU)

- >2024: Mutualized stage. Contribution based on BU

- 2020. COVID-19. EBA impact study:

- + banking risk, + volatility in financial markets - asset quality 


\section{Mutualized stage:}

- EDIS:

- Only deposit insurance in Eurozone. Replace national schemes

- Absorb all liquidity needs and losses

- Problems:

- Cross-border subsidies

- Moral hazard

- Risk-taking behavior

- Solution:

- Risk-sharing methodology for contributions 
- Data sources:

- Orbis Bank Focus database

- European deposit guarantee systems data

- Sample:

- Year: 2018

- Eurozone banks: 806

- Covered deposits: $€ 4.9$ trillions, $81 \%$ in the Eurozone 


\section{Table 1. Representativeness of sample}

\begin{tabular}{|c|c|c|c|c|c|}
\hline Country & $\begin{array}{c}\text { Total } \\
\text { Covered } \\
\text { deposit } \\
\text { population } \\
(\text { bn } €) \\
\end{array}$ & $\begin{array}{c}\text { Covered } \\
\text { deposit } \\
\text { population } \\
(\%)\end{array}$ & $\begin{array}{c}\text { Total } \\
\text { Covered } \\
\text { deposit } \\
\text { sample } \\
(\text { bn } €)\end{array}$ & $\begin{array}{c}\text { Representativenss } \\
\text { of de sample } \\
(\%) \\
\end{array}$ & $\begin{array}{c}\text { Number of } \\
\text { Banks in the } \\
\text { sample }\end{array}$ \\
\hline Austria (AT) & 219 & 3.6 & 179 & 82 & 45 \\
\hline Belgium (BE) & 293 & 4.8 & 275 & 94 & 13 \\
\hline Cyprus (CY) & 26 & 0.4 & 26 & 99 & 18 \\
\hline Germany (DE) & 1815 & 30.0 & 1053 & 58 & 138 \\
\hline Estonia (EE) & 9 & 0.1 & 8 & 87 & 7 \\
\hline Spain (ES) & 726 & 12.0 & 719 & 99 & 27 \\
\hline Finland $(\mathrm{FI})$ & 129 & 2.1 & 125 & 97 & 138 \\
\hline France (FR) & 1168 & 19.3 & 1028 & 88 & 82 \\
\hline Greece (GR) & 104 & 1.7 & 96 & 92 & 5 \\
\hline Ireland (IE) & 106 & 1.8 & 105 & 99 & 7 \\
\hline Italy (IT) & 699 & 11.5 & 601 & 86 & 186 \\
\hline Lithuania (LT) & 14 & 0.2 & 13 & 91 & 5 \\
\hline Luxembourg (LU) & 32 & 0.5 & 17 & 54 & 17 \\
\hline Latvia (LV) & 8 & 0.1 & 8 & 95 & 10 \\
\hline Malta (MT) & 12 & 0.2 & 10 & 86 & 6 \\
\hline Netherland (NL) & 499 & 8.2 & 494 & 99 & 15 \\
\hline Portugal (PT) & 144 & 2.4 & 108 & 75 & 70 \\
\hline Slovenia (SI) & 19 & 0.3 & 16 & 85 & 9 \\
\hline Slovakia (SK) & 32 & 0.5 & 30 & 93 & 8 \\
\hline Total & 6,056 & 100.0 & 4,913 & 81 & 806 \\
\hline
\end{tabular}

Gómez Fernández-Aguado, Pilar, Trigo Martínez, Eduardo, More Evaluation of European Deposit Insurance Scheme Funding Base 
- We used SYMBOL microsimulation model (De'Lisa'2011)

- SYMBOL's methodological phases:

- Step 1. Estimation of the implied obligor probability of default $\left(I O P D_{i}\right)$

- Step 2. Simulation of correlated losses

- Step 3. Determination of bank failure

- Step 4. EDIS loss distribution 
- We analyse contagion risk using three correlations structures:

- $\Sigma_{1}: 1 ; 0.5 ; 0$

- $\Sigma_{2}: 1 ; 0.5 ; 0,5$

- $\Sigma_{3}: 1 ; 0.6 ; 0.3$

- We evaluated model risk using different decomposition methods and calculations procedures

- We run several numbers of simulations:

- 100,000

- 500,000

- $1,000,000$ 
- We use two risk measures:
- VaR
- ES

- and several confidence levels:

- $99 \%$

- $99.5 \%$

- $99.9 \%$

- $99.95 \%$

- $100 \%$

- We conduct a sensitivity analysis of risk portfolio:
- $x 2$
- $\times 5$ 


\section{Table 2. EDIS loss distribution}

\begin{tabular}{|c|c|c|c|c|c|c|c|c|c|}
\hline & \multicolumn{3}{|c|}{$\sum_{1}$} & \multicolumn{3}{|c|}{$\sum_{2}$} & \multicolumn{3}{|c|}{$\sum_{3}$} \\
\hline Defaults & \multicolumn{3}{|c|}{11,124} & \multicolumn{3}{|c|}{10,806} & \multicolumn{3}{|c|}{11,214} \\
\hline Mean (bn $€$ ) & \multicolumn{3}{|c|}{0.78} & \multicolumn{3}{|c|}{0.79} & \multicolumn{3}{|c|}{0.73} \\
\hline St. Dev. (bn $€$ ) & \multicolumn{3}{|c|}{3.89} & \multicolumn{3}{|c|}{3.78} & \multicolumn{3}{|c|}{3.41} \\
\hline Skewness & \multicolumn{3}{|c|}{0.16} & \multicolumn{3}{|c|}{0.15} & \multicolumn{3}{|c|}{0.15} \\
\hline Kurtosis & \multicolumn{3}{|c|}{28.22} & \multicolumn{3}{|c|}{28.20} & \multicolumn{3}{|c|}{26.24} \\
\hline TFCL (\%) & \multicolumn{3}{|c|}{99.97} & \multicolumn{3}{|c|}{99.97} & \multicolumn{3}{|c|}{99.97} \\
\hline Percentile (\%) & $\begin{array}{c}\mathrm{VaR} \\
(\mathrm{b} \in €)\end{array}$ & $\begin{array}{c}\text { ES } \\
(b n €)\end{array}$ & $\begin{array}{l}\mathrm{FN} \\
(\%)\end{array}$ & $\begin{array}{c}\mathrm{VaR} \\
(\mathrm{bn} €)\end{array}$ & $\begin{array}{c}\text { ES } \\
(\mathrm{bn} €)\end{array}$ & $\begin{array}{l}\text { FN } \\
(\%)\end{array}$ & $\begin{array}{c}\mathrm{VaR} \\
(\mathrm{bn} €)\end{array}$ & $\begin{array}{c}\text { ES } \\
(b n €)\end{array}$ & $\begin{array}{l}\text { FN } \\
(\%)\end{array}$ \\
\hline 99.00 & 0.00 & 7.81 & 0.00 & 0.00 & 8.12 & 0.00 & 0.00 & 8.43 & 0.00 \\
\hline 99.50 & 0.00 & 15.63 & 0.00 & 0.00 & 16.24 & 0.00 & 0.00 & 16.87 & 0.00 \\
\hline 99.90 & 0.97 & 77.57 & 0.02 & 0.97 & 80.69 & 0.02 & 0.83 & 83.95 & 0.02 \\
\hline 99.95 & 6.74 & 152.37 & 0.14 & 6.34 & 158.92 & 0.13 & 4.92 & 165.99 & 0.10 \\
\hline 99.96 & 12.60 & 188.34 & 0.26 & 12.32 & 196.42 & 0.25 & 11.13 & 205.71 & 0.23 \\
\hline 99.97 & 23.49 & 244.93 & 0.48 & 22.50 & 255.94 & 0.46 & 22.48 & 268.76 & 0.46 \\
\hline 99.98 & 56.21 & 347.63 & 1.14 & 59.00 & 365.33 & 1.20 & 58.98 & 385.22 & 1.20 \\
\hline 99.99 & 156.78 & 599.75 & 3.19 & 167.21 & 633.17 & 3.40 & 222.29 & 667.83 & 4.52 \\
\hline 100.00 & $1,954.96$ & $1,954.96$ & 39.79 & $2,112.34$ & $2,112.34$ & 43.00 & $2,205.49$ & $2,205.49$ & 44.89 \\
\hline
\end{tabular}

Source: Own work

Gómez Fernández-Aguado, Pilar, Trigo Martínez, Eduardo, More Evaluation of European Deposit Insurance Scheme Funding Base 


\section{Table 3. IOPDx2 sensitivity analysis}

\begin{tabular}{|c|c|c|c|c|c|c|c|c|c|}
\hline & \multicolumn{3}{|c|}{$\sum_{1}$} & \multicolumn{3}{|c|}{$\sum_{2}$} & \multicolumn{3}{|c|}{$\sum_{3}$} \\
\hline Defaults & \multicolumn{3}{|c|}{48,939} & \multicolumn{3}{|c|}{48,257} & \multicolumn{3}{|c|}{49,029} \\
\hline Mean (bn $€$ ) & \multicolumn{3}{|c|}{0.88} & \multicolumn{3}{|c|}{0.92} & \multicolumn{3}{|c|}{1.06} \\
\hline St. Dev. $(b n €)$ & \multicolumn{3}{|c|}{3.98} & \multicolumn{3}{|c|}{4.14} & \multicolumn{3}{|c|}{4.86} \\
\hline Skewness & \multicolumn{3}{|c|}{0.07} & \multicolumn{3}{|c|}{0.07} & \multicolumn{3}{|c|}{0.08} \\
\hline Kurtosis & \multicolumn{3}{|c|}{5.74} & \multicolumn{3}{|c|}{6.39} & \multicolumn{3}{|c|}{7.05} \\
\hline TFCL (\%) & \multicolumn{3}{|c|}{99.88} & \multicolumn{3}{|c|}{99.89} & \multicolumn{3}{|c|}{99.89} \\
\hline Percentile (\%) & $\begin{array}{c}\mathrm{VaR} \\
(\mathrm{bn} €)\end{array}$ & $\begin{array}{c}\text { ES } \\
(b n €)\end{array}$ & $\begin{array}{l}\mathrm{FN} \\
(\%)\end{array}$ & $\begin{array}{c}\mathrm{VaR} \\
(\mathrm{bn} €)\end{array}$ & $\begin{array}{c}\text { ES } \\
(b n €)\end{array}$ & $\begin{array}{l}\mathrm{FN} \\
(\%)\end{array}$ & $\begin{array}{c}\mathrm{VaR} \\
(\mathrm{bn} €)\end{array}$ & $\begin{array}{c}\text { ES } \\
(b n €)\end{array}$ & $\begin{array}{l}\mathrm{FN} \\
(\%)\end{array}$ \\
\hline 99.00 & 0.00 & 39.16 & 0.00 & 0.00 & 37.89 & 0.00 & 0.00 & 39.63 & 0.00 \\
\hline 99.50 & 0.70 & 78.04 & 0.01 & 0.67 & 75.52 & 0.01 & 0.44 & 79.15 & 0.01 \\
\hline 99.90 & 53.58 & 360.41 & 1.09 & 52.10 & 349.00 & 1.06 & 49.27 & 373.27 & 1.00 \\
\hline 99.95 & 173.61 & 629.87 & 3.53 & 162.19 & 608.97 & 3.30 & 163.01 & 660.47 & 3.32 \\
\hline 99.96 & 234.29 & 708.18 & 4.77 & 235.69 & 733.63 & 4.80 & 244.57 & 772.58 & 4.98 \\
\hline 99.97 & 336.07 & 843.80 & 6.84 & 385.34 & 874.76 & 7.84 & 394.91 & 924.22 & 8.04 \\
\hline 99.98 & 483.76 & $1,054.38$ & 9.85 & 586.96 & $1,077.14$ & 11.95 & 597.85 & $1,150.90$ & 12.17 \\
\hline 99.99 & 899.41 & $1,385.44$ & 18.31 & 985.37 & $1,410.40$ & 20.06 & $1,040.64$ & $1,513.96$ & 21.18 \\
\hline 100.00 & $2,499.98$ & $2,499.98$ & 50.89 & $2,751.52$ & $2,751.52$ & 56.01 & $3,316.35$ & $3,316.35$ & 67.50 \\
\hline
\end{tabular}

Source: Own work 


\section{Table 4. IOPDx5 sensitivity analysis}

\begin{tabular}{|c|c|c|c|c|c|c|c|c|c|}
\hline & \multicolumn{3}{|c|}{$\sum_{1}$} & \multicolumn{3}{|c|}{$\Sigma_{2}$} & \multicolumn{3}{|c|}{$\sum_{3}$} \\
\hline Defaults & \multicolumn{3}{|c|}{275,851} & \multicolumn{3}{|c|}{274,632} & \multicolumn{3}{|c|}{276,524} \\
\hline Mean (bn $€$ ) & \multicolumn{3}{|c|}{1.25} & \multicolumn{3}{|c|}{1.26} & \multicolumn{3}{|c|}{1.60} \\
\hline St. Dev. $(b n €)$ & \multicolumn{3}{|c|}{4.94} & \multicolumn{3}{|c|}{5.11} & \multicolumn{3}{|c|}{6.48} \\
\hline Skewness & \multicolumn{3}{|c|}{0.03} & \multicolumn{3}{|c|}{0.03} & \multicolumn{3}{|c|}{0.03} \\
\hline Kurtosis & \multicolumn{3}{|c|}{1.11} & \multicolumn{3}{|c|}{1.13} & \multicolumn{3}{|c|}{1.37} \\
\hline TFCL (\%) & \multicolumn{3}{|c|}{99.34} & \multicolumn{3}{|c|}{99.35} & \multicolumn{3}{|c|}{99.42} \\
\hline Percentile (\%) & $\begin{array}{l}\mathrm{VaR} \\
(\mathrm{bn} €)\end{array}$ & $\begin{array}{c}\text { ES } \\
(b n €)\end{array}$ & $\begin{array}{l}\mathrm{FN} \\
(\%)\end{array}$ & $\begin{array}{l}\mathrm{VaR} \\
(\mathrm{bn} €)\end{array}$ & $\begin{array}{c}\text { ES } \\
(b n €)\end{array}$ & $\begin{array}{l}\mathrm{FN} \\
(\%)\end{array}$ & $\begin{array}{l}\text { VaR } \\
(b n €)\end{array}$ & $\begin{array}{c}\text { ES } \\
(b n €)\end{array}$ & $\begin{array}{l}\mathrm{FN} \\
(\%)\end{array}$ \\
\hline 99.00 & 13.90 & 242.45 & 0.28 & 14.22 & 240.79 & 0.29 & 10.79 & 245.17 & 0.22 \\
\hline 99.50 & 67.68 & 451.94 & 1.38 & 67.83 & 448.86 & 1.38 & 62.53 & 464.90 & 1.27 \\
\hline 99.90 & 708.09 & $1,290.55$ & 14.41 & 696.88 & $1,285.99$ & 14.18 & 722.47 & $1,380.01$ & 14.71 \\
\hline 99.95 & $1,245.28$ & $1,622.61$ & 25.35 & $1,278.30$ & $1,620.30$ & 26.02 & $1,309.99$ & $1,766.24$ & 26.66 \\
\hline 99.96 & $1,362.78$ & $1,693.22$ & 27.74 & $1,433.71$ & $1,694.76$ & 29.18 & $1,456.40$ & $1,869.25$ & 29.64 \\
\hline 99.97 & $1,472.73$ & $1,770.74$ & 29.98 & $1,476.32$ & $1,774.49$ & 30.05 & $1,520.59$ & $1,996.72$ & 30.95 \\
\hline 99.98 & $1,562.30$ & $1,897.31$ & 31.80 & $1,568.73$ & $1,901.94$ & 31.93 & $1,692.69$ & $2,193.39$ & 34.45 \\
\hline 99.99 & $1,746.47$ & $2,151.85$ & 35.55 & $1,737.80$ & $2,166.25$ & 35.37 & $2,008.22$ & $2,563.84$ & 40.88 \\
\hline 100.00 & $3,881.91$ & $3,881.91$ & 79.02 & $3,883.59$ & $3,883.59$ & 79.05 & $4,264.09$ & $4,264.09$ & 86.79 \\
\hline
\end{tabular}

Source: Own work 
Figure 1. EDIS loss distribution.
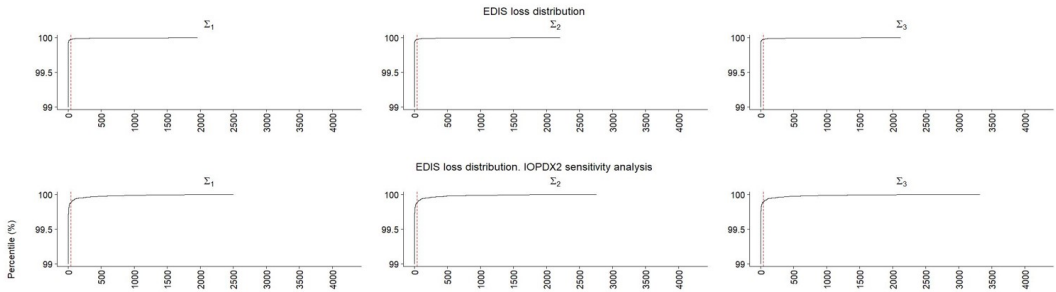

EDIS loss distribution. 1OPDX2 sensitivity analysis
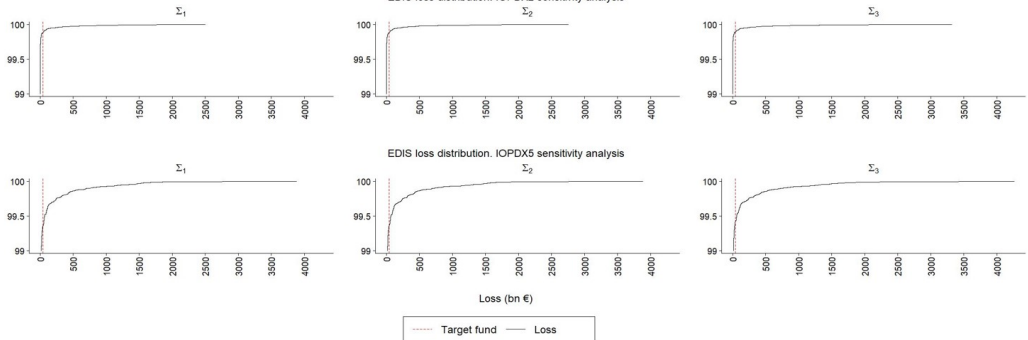

Source: Own work

Gómez Fernández-Aguado, Pilar, Trigo Martínez, Eduardo, More Evaluation of European Deposit Insurance Scheme Funding Base 
- EBA developed a guidelines on methods for calculating risk-adjusted contribution for national DIS (EBA, 2015)

- Member states develop their own calculation methods using established guidelines

- EBA conducts periodic reviews.

- In the last it conclude that no changes are necessary

- Methods to calculate contributions:

- Bucket method

- Sliding scale methods:

- Linear

- Exponential 
Risk-adjusted contribution for i-th bank $\left(C_{i}\right)$ is given for following equation:

$$
C_{i}=C R \cdot A R W_{i} \cdot C D_{i} \cdot \mu
$$

where:

- $C R$ : contribution rate

- $A R W_{i}$ : aggregate risk weight for $i$-th bank

- CDi: covered deposits for $i$-th bank

- $\mu$ : adjustment coefficient 
$A R W$ is determined in the following steps:

(1) Definition of risk indicators (IR)

(2) Transformation of indicator values into an individual risk score (IRS)

(3) Calculation of the aggregate risk score $(A R S)$

(4) Determination of the aggregate risk weight $(A R W)$ 


\section{Table 5. Risk indicators}

\begin{tabular}{|c|c|c|c|c|}
\hline Category & Indicator & Notation & Description & $\begin{array}{c}\text { Expected } \\
\text { sign on } \\
\text { bank risk }\end{array}$ \\
\hline \multirow{4}{*}{$\begin{array}{l}\text { Liquidity and } \\
\text { Funding }\end{array}$} & Leverage ratio & $\mathrm{C} 1$ & Tier 1 capital/Total assets & Negative \\
\hline & Capital coverage ratio & $\mathrm{C} 2$ & $\begin{array}{l}\text { Actual own } \\
\text { funds/Required own } \\
\text { funds }\end{array}$ & Negative \\
\hline & Liquidity ratio & L1 & Liquid assets/Total assets & Negative \\
\hline & Loans-to-deposits ratio & L2 & Loans/Deposit & Positive \\
\hline Asset quality & Non-performing loans ratio & AQ1 & $\begin{array}{l}\text { NPL/Total loans and debt } \\
\text { instruments }\end{array}$ & Positive \\
\hline \multirow{2}{*}{$\begin{array}{l}\text { Business } \\
\text { model and } \\
\text { management }\end{array}$} & $\begin{array}{l}\text { Risk weighted assets (RWA) to } \\
\text { total assets ratio }\end{array}$ & B1 & RWA/Total assets & Positive \\
\hline & Return on assets & B2 & Net Income/Total assets & Negative \\
\hline $\begin{array}{l}\text { Potential } \\
\text { losses for the } \\
\text { DGS }\end{array}$ & $\begin{array}{l}\text { Unencumbered assets/ covered } \\
\text { deposits }\end{array}$ & P1 & $\begin{array}{l}\text { Liquid assets/Covered } \\
\text { deposits }\end{array}$ & Negative \\
\hline
\end{tabular}

Source: Own work 


\section{Table 6. Effect of risk-adjusted contributions in EDIS by country}

\begin{tabular}{|c|c|c|c|c|c|c|c|c|c|c|c|c|}
\hline \multirow[b]{2}{*}{ Country } & \multicolumn{4}{|c|}{ Bucket method } & \multicolumn{4}{|c|}{$\begin{array}{l}\text { Sliding scale method } \\
\text { (linear) }\end{array}$} & \multicolumn{4}{|c|}{$\begin{array}{l}\text { Sliding scale method } \\
\text { (exponential) }\end{array}$} \\
\hline & $\begin{array}{l}\mathrm{ARW} \\
(\%)\end{array}$ & $\begin{array}{l}\mathrm{C}^{\mathrm{EDIS}} \\
(\%)\end{array}$ & $\begin{array}{c}\mathrm{RC}^{\mathrm{EDIS} / \mathrm{DGS}} \\
(\%)\end{array}$ & $\begin{array}{l}\text { LAC } \\
(\%)\end{array}$ & $\begin{array}{l}\mathrm{ARW} \\
(\%)\end{array}$ & $\begin{array}{l}\mathrm{C}^{\mathrm{EDIS}} \\
(\%)\end{array}$ & $\begin{array}{l}\mathrm{RC}^{\mathrm{EDIS} / \mathrm{DGS}} \\
(\%)\end{array}$ & $\begin{array}{c}\mathrm{LAC} \\
(\%)\end{array}$ & $\begin{array}{c}\text { ARW } \\
(\%)\end{array}$ & $\begin{array}{c}\mathrm{C}^{\mathrm{EDIS}} \\
(\%)\end{array}$ & $\begin{array}{c}\mathrm{RC}^{\text {EDISIDGS }} \\
(\%)\end{array}$ & $\begin{array}{l}\text { LAC } \\
(\%)\end{array}$ \\
\hline AT & 106.5 & 0.676 & -15.48 & 99.979 & 96.1 & 0.784 & -1.98 & 99.980 & 74.2 & 0.778 & -2.81 & 99.980 \\
\hline $\mathrm{BE}$ & 114.7 & 0.728 & -8.99 & 99.991 & 95.4 & 0.778 & -2.69 & 99.991 & 73.0 & 0.765 & -4.35 & 99.991 \\
\hline $\mathrm{CY}$ & 107.4 & 0.682 & -14.77 & 99.996 & 87.7 & 0.716 & -10.54 & 99.996 & 68.6 & 0.719 & -10.09 & 99.996 \\
\hline $\mathrm{DE}$ & 122.1 & 0.775 & -3.14 & 99.976 & 92.8 & 0.758 & -5.30 & 99.976 & 71.9 & 0.753 & -5.87 & 99.976 \\
\hline EE & 57.3 & 0.364 & -54.50 & 99.998 & 65.4 & 0.533 & -33.32 & 99.998 & 56.9 & 0.597 & -25.40 & 99.998 \\
\hline ES & 146.3 & 0.929 & 16.07 & 99.976 & 101.9 & 0.832 & 3.97 & 99.975 & 78.6 & 0.824 & 3.02 & 99.975 \\
\hline FI & 81.8 & 0.520 & -35.05 & 99.995 & 91.7 & 0.748 & -6.45 & 99.995 & 71.2 & 0.746 & -6.70 & 99.995 \\
\hline FR & 134.7 & 0.855 & 6.90 & 99.975 & 90.7 & 0.740 & -7.46 & 99.975 & 70.1 & 0.734 & -8.20 & 99.975 \\
\hline GR & 138.4 & 0.879 & 9.88 & 99.995 & 121.2 & 0.989 & 23.65 & 99.995 & 95.4 & 0.999 & 25.03 & 99.995 \\
\hline IE & 123.1 & 0.782 & -2.30 & 99.996 & 115.6 & 0.944 & 17.94 & 99.996 & 89.5 & 0.938 & 17.31 & 99.996 \\
\hline IT & 144.0 & 0.915 & 14.32 & 99.971 & 120.9 & 0.986 & 23.30 & 99.974 & 97.3 & 1.020 & 27.44 & 99.975 \\
\hline $\mathbf{L T}$ & 84.6 & 0.537 & -32.83 & 99.997 & 85.2 & 0.696 & -13.05 & 99.997 & 67.5 & 0.707 & -11.63 & 99.997 \\
\hline LU & 75.4 & 0.479 & -40.14 & 99.987 & 83.3 & 0.680 & -15.01 & 99.994 & 65.8 & 0.690 & -13.81 & 99.994 \\
\hline LV & 83.3 & 0.529 & -33.91 & 99.995 & 84.5 & 0.689 & -13.84 & 99.995 & 67.8 & 0.710 & -11.23 & 99.995 \\
\hline MT & 87.2 & 0.553 & -30.83 & 99.993 & 89.3 & 0.729 & -8.91 & 99.996 & 69.2 & 0.725 & -9.39 & 99.996 \\
\hline $\mathrm{NL}$ & 89.7 & 0.570 & -28.80 & 99.997 & 84.9 & 0.693 & -13.42 & 99.998 & 66.5 & 0.697 & -12.89 & 99.998 \\
\hline PT & 146.7 & 0.932 & 16.45 & 99.983 & 109.4 & 0.893 & 11.63 & 99.983 & 83.9 & 0.879 & 9.88 & 99.983 \\
\hline SI & 128.0 & 0.813 & 1.60 & 99.992 & 109.8 & 0.896 & 12.04 & 99.992 & 84.2 & 0.883 & 10.34 & 99.992 \\
\hline SK & 123.6 & 0.785 & -1.88 & 99.957 & 114.0 & 0.930 & 16.26 & 99.957 & 87.5 & 0.917 & 14.68 & 99.957 \\
\hline
\end{tabular}

Gómez Fernández-Aguado, Pilar, Trigo Martínez, Eduardo, More Evaluation of European Deposit Insurance Scheme Funding Base 
- Consolidation of the BU:

- have enabled greater resilience of banks

- COVID-19 pandemic could trigger a severe scenario

- Development of EDIS

- We present a quantitative analysis of EDIS financing:

- Soundness of deposit insurance

- Clarify disciplinary problems

- We use SYMBOL model to simulate EDIS loss distribution:

- phenomenon is rare but with very high severity

- distribution is skewed and has a very thick tail

- Losses depend on:

- Correlations

- Risk of credit portfolios 
- Cost of insurance varies from the European Deposit Insurance Scheme to national ones

- EDIS:

- Degree of risk aversion/level of solvency

- Equitable risk measures/improve risk management

- Cross-border subsidies 


\section{Thank you for your attention}

This work has been financed by I Plan Propio De Investigación, Transferencia y Divulgación Cientifica, Universidad de Málaga, Campus de Excelencia Internacional Andalucía Tech 